\title{
Marcos de control y estándares para el gobierno de tecnologías de información (TI)
}

Control frameworks and standards for information technology governance (ITG)

\section{FRANCISCO ARNALDO VARGAS-BERMÚDEZ}

Ingeniero de Sistemas

Magíster en Ciencias de la Información y las Comunicaciones Grupo de Investigación Gisdytel Universidad de Boyacá, Colombia franciscovargasb@uniboyaca.edu.co 


\title{
RESUMEN
}

En este artículo se plantea una visión general acerca del gobierno $\mathrm{TI}$, y como éste se relaciona con el gobierno corporativo a través de herramientas que permiten hacer más eficiente y eficaz dicho gobierno. Se hace una breve descripción de los conceptos y de la evolución histórica de algunas herramientas de este tipo, tales como los marcos de control (COBIT, ISO 38500) y las normas (PMBOK, CMMI, ISO 20000, ITIL) que permiten la ejecución de los lineamientos o estrategias diseñadas por dichos marcos, todo ello con el objeto de alinear los objetivos de gobierno de la organización con las Tecnologías de Información.

Palabras clave: COBIT, ISO 38500, Gobierno TI, CMMI, PMBOK, ITIL.

\begin{abstract}
This paper presents an overview about the Information Technology Governance (ITG), and how it is related to the corporate administration (CA) through optimization tools, that allow the ITG to be more efficient and effective. Some concepts and the historical evolution of some tools, such as control frameworks (COBIT, ISO 38500) and standards (PMBOK, CMMI, ISO20000, ITIL) which allow the execution of the guidelines or strategies designed by control frameworks are briefly described, this in order to line up the objectives of the organization with the information technologies.
\end{abstract}

Keywords: COBIT, ISO 38500, IT Governance, CMMI, PMBOK, ITIL. 


\section{INTRODUCCIÓN}

Actualmente las organizaciones poseen un componente tan importante como lo son el financiero, el administrativo y otros; dicho componente son las Tecnologías de Información (TI), las cuales permiten el funcionamiento óptimo de la organización. El desconocimiento de las TI por parte de la mayoría de las áreas que componen la organización, conlleva a que no se valore la importancia de ésta y por ende se aísle sin aprovechar las ventajas competitivas que dichas TI ofrecen. Se observa que el problema es la falta de relación entre los objetivos corporativos y los objetivos de la TI, es aquí donde aparece el gobierno TI, permitiendo realizar una alineación entre dichos objetivos, a través de la definición de marcos de control y normas encaminadas a la ejecución de éstos; definición realizada por la academia, por agencias de investigación, por entidades de estándares, entre otras.

En este artículo se hace una breve descripción de conceptos tales como gobierno Tl, marcos de gobierno, relación entre gobierno corporativo y gobierno TI. Se presentan algunos marcos de control junto con las normas que permiten la ejecución de las políticas estratégicas definidas en dichos marcos, y por último se plantea un análisis de la temática y algunas conclusiones.

\section{GOBIERNO TI}

Es de resaltar la importancia que han tenido las tecnologías de información (comunicaciones y sistemas de información) en la forma de gestionar las empresas actualmente; han impactado en la forma de gobernarla, en el área operativa, financiera e incluso en la propia área de TI.

A continuación se desarrollará una breve descripción de la evolución que ha tenido en los últimos años el proceso de gestión de TI dentro de las organizaciones, iniciando con la conceptualización necesaria para un buen entendimiento de dicha temática (Fernández y Llorens, 2005).

\section{Concepto}

Es importante resaltar la relación directa que existe entre gobierno corporativo y gobierno TI, debido a la convergencia de éstos en la actividad de gestión encaminada al cumplimiento de la visión organizacional. En la Figura 1 se puede observar dicha relación de forma clara, donde la transición desde el gobierno corporativo hacia el gobierno de TI recorre caminos que involucra a equipos ejecutivos, al área de información y TI, a través de estrategias y comportamientos deseables. 


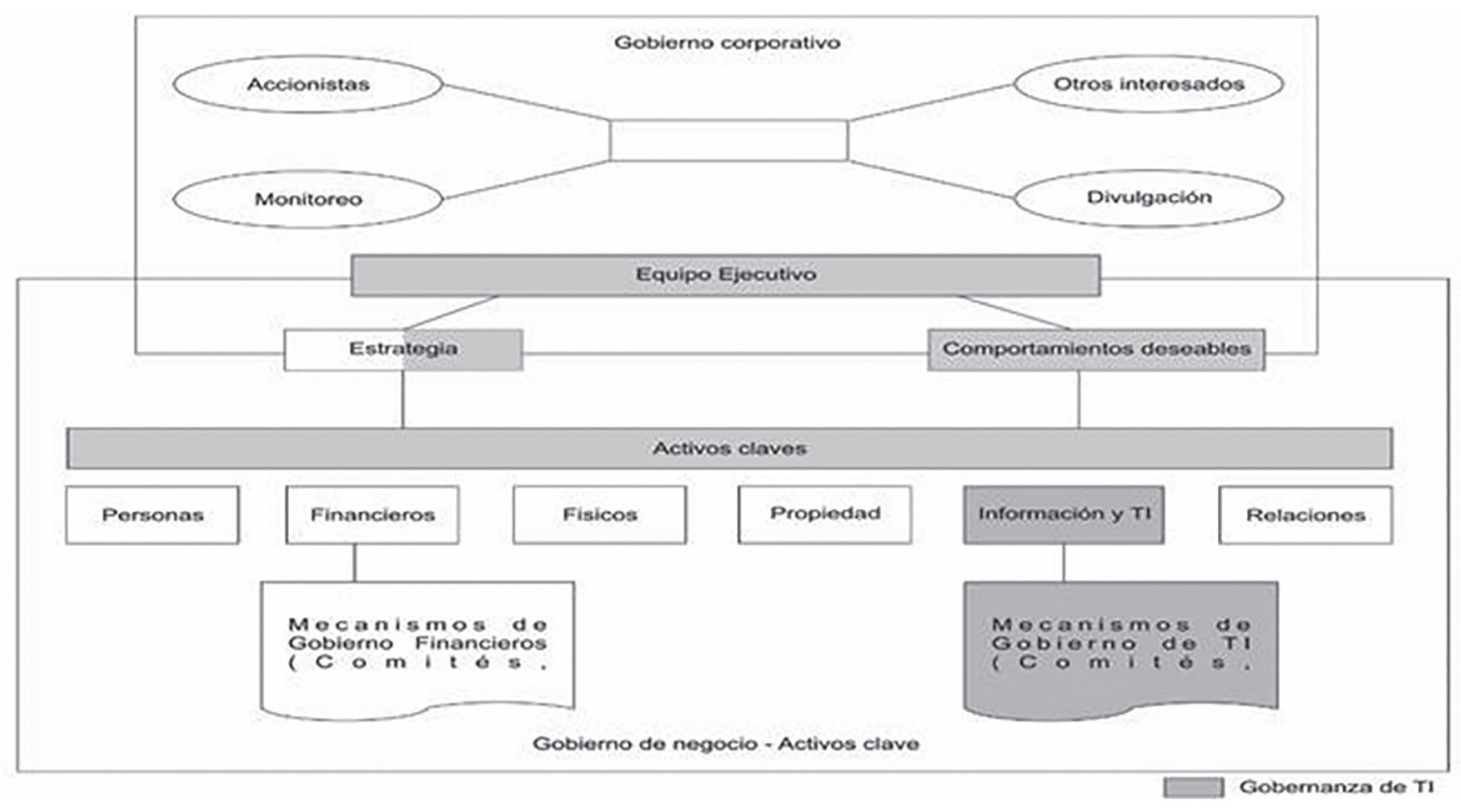

Figura 1. Modelo de Peter Weill y Jeane W. Ross Fuente: (Weill y Ross, 2004).

Son variados los conceptos que existen a cerca de gobierno TI, cada uno enfocado según la óptica del autor pero con algo en común: gestión y tecnologías de información. En éstos se coincide puesto que básicamente son la razón de ser de dicho proceso. Algunas de estas definiciones, son:

Definición 1. "Es un marco para la toma de decisiones y la asignación de responsabilidades para facilitar el resultado deseado respecto al uso de la TI" (Zamora, 2010).

Definición 2. "Responsabilidad de los ejecutivos y del consejo de directores; parte integral del Gobierno Corporativo que consta de liderazgo, estructuras organizacionales y procesos que garantizan que la TI de la empresa soportará y extenderá las estrategias y objetivos organizacionales" (Forrester firma de Consultores y Craig S, 2005).

Pero ¿cómo se logra un equilibrio entre los recursos de la organización, las plataformas de tecnologías de información con que se cuenta o que se requieren y cómo lograr gestionar todos los componentes que convergen en este proceso? para ello se ha creado una serie de marcos de control y normas que permiten hacer tangible los lineamientos trazados por dichos marcos; a continuación se hará un tratamiento general de los más relevantes. 


\section{Marcos de gobierno TI}

Es de vital importancia direccionar los objetivos del negocio con el soporte que dará la TI a la obtención de éstos; ello a través del diseño de planes de acción, cuya ejecución requiere las TI y el control de riesgos que esto implica. Con el objeto de lograr una relación armoniosa entre estos componentes se hace necesario el diseño de marcos que permitan estructurar y operacionalizar todo este andamiaje, es por ello que algunos de estos (COBIT, ISO 38500) serán tratados en el presente artículo de forma general.

\section{Objetivos de control para la información y la tecnología relacionada (Control Objectives for Infor- mation and Related Technology - COBIT)}

Ha sido creado por ITGI (ITGI, 2007; ITGI, 2008), experimentando una evolución a través de un conjunto de adecuaciones y mejoras, pasando por COBIT 4.1 hasta llegar a COBIT 5 (ISACA, 2011; OGC, 2008); la siguiente descripción será enfocada en la versión 4.1 puesto que la 5 se encuentra en evaluación, es decir, no ha sido formalizado su uso.

La aplicación de COBIT permitirá la optimización de las inversiones realizadas en TI, asegurar la entrega del servicio y definir una medida como referencia para conocer el estado del proceso. El uso de este permite asegurar que la TI satisfaga los requerimientos del negocio.

COBIT establece que es responsabilidad de la gerencia salvaguardar todos los activos de la empresa. Para descargar esta responsabilidad, así como para lograr sus expectativas, la gerencia debe establecer un adecuado sistema de control interno. COBIT proporciona un conjunto detallado de controles y de técnicas de control para el entorno de administración/gestión de sistemas de información (ISACA, 2005).

Algunas de las características más relevantes de este marco de trabajo son: orientado a negocios, orientado a procesos, basado en controles e impulsado por mediciones.

a. Orientado al negocio (ITGI, 2008a). Es el enfoque más importante para COBIT. Está diseñado para ser el soporte de los gerentes y los dueños de los procesos de negocio, así como también para proveedores de servicios, usuarios y auditores de TI.

La Figura 2 permite observar el principio básico de COBIT, el cual define que para lograr satisfacer los requerimientos del negocio y con ello alcanzar los objetivos de éste, es necesario involucrar las TI, desarrollando una administración y control adecuados de la misma. 


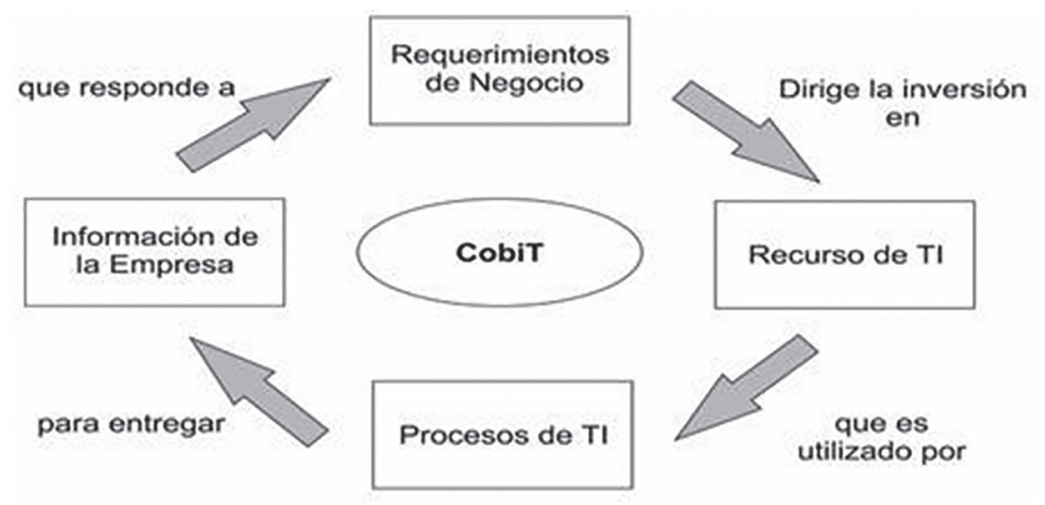

Figura 2. Principio básico de COBIT.

Fuente: (ISACA, 2005)

b. Orientado a procesos. Permite definir las responsabilidades de cada miembro de la organización, las actividades y los riesgos que deben ser gestionados. Para ello se han clasificado en 4 dominios:

Planear y Organizar (PO) el cual proporciona dirección para la entrega de soluciones y la entrega de servicios, Adquirir e implementar (AI) este proporciona las soluciones y las pasa para convertirlas en servicios, Entregar y dar Soporte (DS) recibe soluciones y las hace utilizables por los usuarios finales, Monitorear y Evaluar (ME) monitorea todos los procesos para asegurar que se sigue la dirección provista (ITGI, 2007).

COBIT ha identificado dentro de estos 4 dominios, 34 procesos de TI, cada uno de los cuales tiene un enlace a las metas de negocio y TI que soporta.

c. Basado en Controles (ITGI, 2007). Si se desea ejecutar un gobierno efectivo, se debe implementar los controles necesarios dentro de un marco de control definido para todos los procesos TI. Cada uno de los 34 procesos TI tiene un objetivo de control de alto nivel y varios objetivos de control detallados. Estos últimos se identifican por dos caracteres que representan el dominio (PO, AI, DS, ME) más un número de proceso y un número de objetivo de control.

d. Impulsado por la medición. Autoevaluarse de forma objetiva no es una tarea fácil, es por ello que COBIT sugiere una serie de herramientas que permitirá a las empresas medir dónde se encuentran y dónde se requieren mejoras; dichas herramientas son: Modelo de madurez, metas y mediciones de desempeño para los procesos de TI y metas de actividades para facilitar el desempeño efectivo de los procesos. 


\section{ISO 38500 Corporate Governance of Information Technology}

Se basa en la norma Australiana AS8015:2005 y fue publicado en junio de 2008. El objetivo de esta norma es proveer una estructura de principios a los directores de las organizaciones, la cual puede ser utilizada en la evaluación, dirección y supervisión del uso de las TI en sus organizaciones. Entre los principios que define para ofrecer un buen gobierno de TI se encuentran: Responsabilidad, estrategia, adquisición, rendimiento, conformidad y conducta humana (ISO/IEC, 2008, Sylvester, 2011).

Este modelo se fundamenta en tres tareas principales: evaluar el uso actual y futuro de las TI, preparación directa e implementación de planes y políticas para garantizar que el uso de las TI cumplen los objetivos de negocio y monitorear la relación entre políticas y desempeño de los planes, las cuales permiten la dirección y control del desempeño de los diferentes roles que convergen en la gestión de la organización para la planificación, implementación y utilización operacional de TI (Toomey, 2009).

Este estándar es apoyado por COBIT, a través de la interrelación de sus 34 procesos con los principios de ISO 38500, lo cual permite observar el aprovechamiento de las fortalezas que cada uno de los modelos de gobierno TI ofrecen, esto debido a que ninguno de los marcos provee un conjunto completo de gobierno TI, todo ello converge en la definición de estrategias más eficientes y eficaces en pro de la buena gestión de las TI.

\section{NORMAS Y ESTÁNDARES DE GOBIERNO TI}

\section{PMBok}

Existe una gran diversidad en la naturaleza de los proyectos a desarrollar en las diferentes áreas del quehacer humano, es por ello que un grupo de profesionales en el año 1969 fundaron lo que hoy se conoce como Instituto de Gerencia de Proyectos (PMI) (PMI, 2012; PMI, 2008), el cual es una asociación profesional sin ánimo de lucro, que se encarga de reunir las mejores prácticas a nivel mundial en gerencia de proyectos, y con ellas construir una guía conocida como PMBok, donde se explican e ilustran éstas. Según PMBok (Bueno, 2010), los siguientes son conceptos básicos a tener en cuenta para aplicar la metodología:

- Un proyecto es un esfuerzo temporal que se lleva a cabo para crear un producto, servicio o resultado único (Paliza, 2009).

- La dirección de proyectos es la aplicación de conocimientos, habilidades, herramientas y técnicas a las actividades del proyecto para cumplir con los requisitos del mismo (PMI, 2008, p. 12). 
Las organizaciones diseñan su plan estratégico (Serna, 1997) con el ánimo de definir el direccionamiento estratégico, el diagnóstico estratégico, las estrategias y la formulación de proyectos, planteando en este último una serie de proyectos que permitan hacer tangibles las estrategias definidas para ejecutar la misión y alcanzar la visión de la empresa. Es por ello que algunos otros conceptos importantes y que convergen en esta disciplina son: la dirección de programas (PMI, 2008, p. 15) y la gestión de portafolios (PMI, 2008, p. 14).

La guía consta de los 42 procesos de la dirección de proyectos; los cuales se agrupan en 5 grandes procesos (Sparano, 2011): Initiating processes, planing processes, closing processes, executing processes y monitoring \& controlling processes (PMI, 2008, 43). Estos procesos se vinculan entre sí a través de los resultados que producen, es decir, las salidas de unos procesos son las entradas de otros, por ejemplo: El grupo de procesos de planificación entrega el plan para la dirección del proyecto y los documentos del proyecto al grupo de procesos de ejecución.

Además de estos procesos, la guía ha sido estructurada en 9 áreas de conocimiento (González, 2010; Sparano, 2011) las cuales se pueden observar en Paliza y Olivera, 2010. Estas buscan cubrir los diferentes frentes que se involucran en un proyecto tales como el tiempo, los costes, calidad del proyecto, recurso humano, riesgos y algunos otros. Dentro de cada una de ellas se observa los diferentes procesos y actividades que las conforman, donde a su vez, cada proceso tiene entradas, salidas y unas herramientas y técnicas que permitirán realizar el manejo de las entradas y la obtención de salidas elaboradas.

\section{CMMI (Capability Maturity Model Integration)}

El Instituto de Ingeniería de Software (Software Engineering Institute) conocido como SEI (SEI, 2012a), es el que creó y mantiene el modelo de calidad CMM - CMMI (Gracia, 2005; ISACA, 2012). La primera versión de CMM tiene un enfoque netamente al área de software; experimentando muchas modificaciones hasta llegar a tener en cuenta otras dos áreas además del software, las cuales son Adquisición (SEI, 2012c) y Servicios (SEI, 2012d). El CMM - CMMI es un modelo de calidad del software que clasifica las empresas en niveles de madurez. Estos niveles sirven para conocer la madurez de los procesos que se realizan para producir software (Gracia, 2005).

¿A qué se hace referencia cuando se habla de madurez? al grado de mejora continua que se realiza en un proceso respecto a un estado. Trasladando dicho concepto al tema de madurez de un proceso de software, el cual hace referencia a un proceso específico que está explícitamente definido, administrado, medido, controlado y es efectivo, se puede decir que una organización adopta 2 estados (inmadura, madura) en cuanto a la madurez de los procesos de software. 
Como CMMI busca la mejora de los procesos de software, define prácticas que se publican en documentos llamados modelos. Tal cómo se expresó anteriormente, en la actualidad existen dos áreas de interés nuevas cubiertas por dichos modelos de CMM, las cuales son: Desarrollo y Adquisición. El modelo CMMI define dos formas de organización, el escalonado y el continuo el uso de cada uno de ellos depende de la organización, de acuerdo a sus características y prioridades de mejora.

El modelo escalonado establece un conjunto de prácticas agrupadas en Áreas Clave de Proceso, las cuales a su vez se agrupan en cinco niveles de madurez: Inicial (CMMI, 2006), Repetible (Palazzolo, 2008), Definido (Palazzolo, 2008), Gestionado (CMMI, 2010), Optimizado (CMMI, 2010); la organización que posea las prácticas incluidas en un nivel y sus inferiores, se considera que ha alcanzado ese nivel de madurez.

\section{ISO 20000}

En mayo de 2005 la ISO (International Organization for Standards) y la IEC (International Electrotechnical Commission), aprobaron la creación de ISO 20000, la cual se basó en la norma británica llamada BS15000 publicada en el año 2000, norma que a su vez está fuertemente ligada al modelo ITIL (IT Infrastructure Library), situación que hace a ISO 20000 totalmente compatible con ITIL. 3.1 (Martínez, 2010; Aeonor, 2009). Este nuevo estándar promueve la adopción de un modelo de procesos integrados destinado a mejorar la eficacia en la prestación de los servicios tecnológicos y establece las directrices para una gestión de servicios de TI de calidad (Turbitt, 2006).

ISO/IEC 20000:2005 está dividida en dos partes bajo el título Gestión de Servicio de Tecnología de la Información:

- ISO/IEC 20000:1:2005 (Parte 1): Se fundamenta en los lineamientos teóricos que se deben seguir con el ánimo de gestionar los servicios de TI.

- ISO/IEC 20000:2:2005 (Parte 2): Se enfoca en describir las mejores prácticas para la gestión de los servicios TI, basadas en lo definido en la parte 1 .

Es importante resaltar que esta norma es la primera en permitir la certificación a nivel mundial de un sistema de gestión de servicios de TI.

\section{ITIL (Information Technology Infraestructure Library)}

Las empresas continuamente requieren más tecnología para optimizar su funcionamiento, esto ha generado la necesidad de servicios informáticos de calidad que se correspondan con los objetivos del negocio, con el ánimo de satisfacer las necesidades del cliente. Todo ello implica la gestión de los 
servicios TI, lo cual se realiza a través de la biblioteca de infraestructura de tecnologías de información (ITIL) (Rodríguez y García, 2008). ITIL es un conjunto de conceptos y de buenas prácticas para la entrega de servicios TI hacia las operaciones de TI (Gatti, 2009; Osiatis, 2010; Phuc, 2011; Pink, 2004).

ITIL fue establecido por la oficina de comercio del gobierno del Reino Unido (OGC), creando una serie de libros donde definía los lineamientos para la gestión de servicios TI. El número de libros en sus primeras versiones era de más de treinta, así fue evolucionando hasta sintetizarse en 5 libros (ITIL service strategy, ITIL service design, ITIL Service Transition, ITIL Service Operation, ITIL Continual Service Improvement) que estructuran la versión 3.

Se inicia con la versión 1 en el año 1996, la cual es analizada y estructurada dando paso a la versión 2 oficializada en el año 2000, en el año 2007 se pública la versión 3 y la edición actual es publicada en el 2011, la cual presenta numerosas aclaraciones en los 5 libros, especialmente en el libro Service Strategy haciéndolo mucho más fácil de leer y más práctico (ItSMF, 2012).

Algunos autores consideran a ITIL como un marco de gobierno y otros tienen un concepto más modesto a cerca de éste, considerándolo como un estándar que apoya al gobierno TI. De hecho ITIL tiene correlación con algunos marcos de gobierno, entre ellos se puede citar a COBIT.

Así como los anteriores existen algunos otros estándares también muy útiles para el desarrollo de estos marcos, entre ellos se puede mencionar a: BS 25999 (BSI, 2012), PCI DSS (PCI, 2010), ISO 27002 (ISO, 2011).

\section{CONSIDERACIONES FINALES}

Como se ha expuesto anteriormente se observa que el gobierno TI es uno de los componentes con mayor impacto en la alineación de los objetivos del negocio con los de TI.

La gerencia define un direccionamiento estratégico que indica hacia donde debe dirigirse la empresa; lo cual implica el trabajo mancomunado de las diferentes áreas o dependencias que componen la organización, trabajo que debe ser apoyado, soportado y facilitado por una plataforma de TI que permita el desarrollo de las labores propias de la misión institucional; en el desarrollo de estas se presentarán situaciones en las cuales se necesitará información de diferente índole: estados financieros, recurso humano, administrativa, y así como esta mucha más información. Dicha información puede ser adquirida, procesada de forma eficiente y eficaz gracias a las bondades de una serie de instrumentos tecnológicos y es vital para el buen funcionamiento de las organizaciones, debido a la necesidad de toma de decisiones por parte de las directivas y los accionistas de estas; decisiones que impactan para bien o para mal el desarrollo de las organizaciones. 
Por lo anterior, una buena gestión de las TI en las empresas adquiere bastante importancia y relevancia, de allí que se adopten los diferentes marcos de gobierno tales como COBIT, ISO 38500, entre otros, con el objeto de definir y trazar planes estratégicos que permitan la administración de éstas con el ánimo de aprovecharlas al máximo y alcanzar un excelente desarrollo de la organización. Para hacer tangibles estos planes estratégicos es importante el uso de una serie de normas tales como PMBok, CMMI, ITIL, ISO 20000, entre otras, que permiten hacer operativo o llevar a la práctica todas las estrategias definidas en esta dirección.

\section{CONCLUSIONES}

PMBok, CMMI, ITIL, ISO 20000 permiten ejecutar y hacer tangible buenas prácticas en el uso de las TI, lo cual debe ser aprovechado, a través de la interrelación de las mismas, con el objeto de crear estrategias que permitan potenciar las TI desde las diferentes ópticas.

En nuestro ámbito es importante diseminar el conocimiento acerca de los marcos de gobierno TI y las normas que permiten la implementación del "cómo" de un "qué" a partir de los marcos de gobierno TI, puesto que ello permitirá el desarrollo de la economía regional basada en la buena gestión de las $\mathrm{TI}$, a nivel empresarial.

En la actualidad las TI se han convertido en un recurso tan importante como lo son las finanzas, el talento humano, lo administrativo, entre otros; es por ello que gestionarlas de una forma adecuada es de vital importancia para el buen funcionamiento y éxito de las organizaciones, afortunadamente existen los marcos de gobierno TI y las normas de referencia, que hacen dicha gestión posible; para ello debe tenerse en cuenta el marco y normas que más se ajuste a las características de la empresa.

El proceso de gobierno de TI es un proyecto a largo plazo, implicando un arduo trabajo por parte de los funcionarios involucrados en el área de TI, de los directivos, de los administrativos y de los clientes.

Es importantísimo que exista una alineación del gobierno corporativo con el gobierno de TI, lo cual se logra identificando los puntos principales donde se desarrolle articulaciones entre éstos.

Lo más importante es cumplir con las cinco áreas focales (Alineamiento estratégico, Entrega de valor, Administración de riesgos, Administración de recursos y Medición del desempeño) del gobierno de $\mathrm{TI}$, sin importar que marco de control se utilice. 


\section{REFERENCIAS BIBLIOGRÁFICAS}

Albero, C. (2009). Modelo de Desarrollo para Gobierno TI. Scientia Et Technica, ISSN: 0122-1701. Universidad Tecnológica de Pereira. Recuperado el 08/02/2012 en: http://www.doaj.org/doaj?func=fulltext\&passMe $=$ http://redalyc.uaemex.mx/src/inicio/ArtPdfRed.jsp?iCve $=84916680032$

Alonso Almeida, M. (2007). El gobierno corporativo electrónico: Análisis desde el enfoque de confianza. ISBN: 978-84-9821-854-1. España. Recuperado el 09/02/2012 en: http://ialnet.unirioja.es/ servlet/libro?codigo $=490653$

Asociación Española de Normalización y Certificación. (2009). ISO/IEC 20000. Guía completa de aplicación para la gestión de los servicios de tecnologías de la información. Aenor Ediciones.

Axentia. (2006). Una introducción a CMMI. (pp. 1-56).

British Standards Institution. (2012). BS 25999 Business continuity. Recuperado el 30/04/2012 en: http://www.bsigroup.com/en/Assessment-and-certification-services/management-systems/Standards-and-Schemes/BS-25999/

Bueno Rabadán, F. (2010). Fundamentos de gestión de proyectos para la preparación del examen de certificación PMP.

Calder, A. (2008). The Calder-Moir ITGovernance Framework. Recuperado el 19/01/2012 en: http:// www.itgovernance.co.uk/calder_moir.aspx

CMMI Product Development Team. (2006). CMMI for Development. Version 1.2 Pittsburgh, A: Software Engineering Institute. Carnegie Mellon University

Fernandez, A. And Llorens, F. (2005). An IT Governance Framework for Universities in Spain. Recuperado el 16/04/2012 en: http://rua.ua.es/dspace/bitstream/10045/11216/1/EUNIS\%202009\%20 -\%20An\%20IT\%20Governance $\% 20$ Framework\%20for\%20Universities\%20in\%20Spain $\% 20-\% 20$ Fernandez\%20y\%20Llorens.pdf

Forrester firma de Consultores y Craig S. (March 2005). IT Governance Framework. Forrester ${ }^{\circledR}$ Best Practices, Craig Simons.

Garbarino, H. (2010). Gobierno de TI. Organización, administración y control de las TI, un encuadre en Pymes. (p. 121). Recuperado el 20/01/2012 en: http://www.ort.edu.uy/fi/pdf/investigaciontuteladagarbarinoort.pdf

Gatti, F. (2009). ITIL-V3. Tata Consultancy Services. 
Globales. (2010). Capability Maturity Model Integration. Recuperado el 18/02/2012 en: http://www. globales.es/imagen/internet/Informaci\%C3\%B3n\%20General\%20CMMI.pdf

González, J (2010). Las Métricas. Recuperado el 21/05/2011en: http://www.liderdeproyecto.com/videoboletin/video008a.html

Gracia J. (2005). CMM - CMMI. Bogotá. Recuperado el 15/02/2012 en: http://www.ingenierosoftware. com/calidad/cmm-cmmi.php

Institute Engineering Software. (2012). CMMI | CMMI Solutions | CMMI for Development. Recuperado el 24/04/2012 en: http://www.sei.cmu.edu/cmmi/solutions/dev/

Institute Engineering Software. (2012). CMMI-ACQ Comparisons. Recuperado el 24/04/2012 en: http:// www.sei.cmu.edu/cmmi/solutions/acq/comparisons.cfm

Institute Engineering Software. (2012). CMMI-SVC Comparisons. Recuperado el 24/04/2012 en: http:// www.sei.cmu.edu/cmmi/solutions/svc/comparisons.cfm

Institute Engineering Software. (2012). CMMI for Development: Guidelines for Process Integration and Product Improvement, Third Edition. Recuperado el 27/04/2012 en: http:/www.sei.cmu.edu/ library/abstracts/books/0321711505.cfm

Institute Engineering Software. (2012). CMMI Version 1.3 Information Center. Recuperado el 30/04/2012 en: http://www.sei.cmu.edu/cmmi/solutions/info-center.cfm

Isaca. (2005). Information Systems Audit and Control Association. Estándar de auditoría de SI gobernabilidad de TI documento s10. (2005). publicación. Recuperado el 29/11/2011 en http://www. isaca.org/Knowledge-Center/Standards/Documents/Standards-IT-Spanish-S10.pdf

Isaca. (2009). IT Value. Recuperado el 10/03/2012 en: http://www.isaca.org/Knowledge-Center/Val-ITIT-Value-Delivery-/Documents/IT-Value-Special-Compilation.pdf

Isaca. (2011). COBIT 5: The Framework. Recuperado el 30/11/2011 en: http://www.isaca.org/Knowledge-Center/Research/Documents/COBIT5-Framework-ED-27June2011.pdf

Isaca. (2012). JOnline: Auditing CMMI Maturity and Sarbanes-Oxley Compliance. Recuperado el 02/05/2012 en http://www.isaca.org/Journal/Past-Issues/2007/Volume-3/Pages/JOnline-Auditing-CMMI-Maturity-and-Sarbanes-Oxley-Compliance.aspx

ISO/IEC. (2008). ISO/IEC 38500 Corporate governance of information technology

ISO. (2011). ISO Code of Practice for Information Security Management (ISO 17799, 27002). Recuperado el 02/05/2012 en: http://privacy.med.miami.edu/glossary/xd_iso_27002_index.htm 
IT governance institute. (2007). COBIT 4.1. Rolling Meadows, IL: Autor.

IT governance institute. (2008). Governance of IT Investments Getting Started With Value Management. Recuperado el 23/03/2012 en: http://www.isaca.org/Knowledge-Center/Val-IT-IT-Value-Delivery-/Documents/Val-IT-Getting-Started-Jul-2008.pdf

ITGI y OGC. (2008). Alineando COBIT 4.1, ITIL V3 e ISO/IEC 27002 en beneficio del negocio. Estados Unidos.

ITGI. (2012). The CALDER-MOIR IT Governance Framework. Recuperado el 12/04/2012 en: http://www. itgovernance.co.uk/calder_moir.aspx

itSMF. (2012). Service Talk la revista del foro de la gestión del servicio TI. Edición I. pp. 25-26.

Martínez, B. (2010). Moving towards ISO 20000. Recuperado el 28/05/2012 en: http://www.rediris.es/ difusion/publicaciones/boletin/88-89/Ponencia5.B.pdf

Morales F. M. (2012). Cuáles son las novedades del PMBOK Guide 5th Edition. Universidad de los Andes. Recuperado el 19/04/2012 en: http://www.projectical.com.co/

Osiatis. (2010). ITIL V3 Gestión de Servicios. Recuperado el 29/04/2012 en: http://ITILv3.osiatis.es/ gestion_servicios_ti.php

Palazzolo, C. (2008). Herramientas y Procesos de Software

Paliza Morales, Alex y Olivera Peñarrieta, Mayra. (2010). Gerencia de proyectos (PMI). (p. 15).

Paliza Morales, A., Peñarrieta, M.A. (2009). Gerencia de Proyectos PMI. (pp. 3). Universidad Andina del Cusco.

PCI Security Standards Council. (2010). PCI SSC Data Security Standards Overview. Recuperado el 30/04/2012 en: https://www.pcisecuritystandards.org/security_standards/index.php

Pink. (2004). The ITIL Story White Paper. Recuperado el 29/04/2012 en: http://jdcmg.isc.ucsb.edu/docs/ ITIL/ITIL-The_Story_of_ITIL.pdf

PMI. (2008). Guía de los fundamentos para la Dirección de Proyectos (Guía del PMBOK). Cuarta edición. Newtown Square, Pennsylvania.

Project Management Institute. (2012). Making project management indispensable for business results.

Rodríguez, R, García, L. (2008). La Gestión de los Procesos de Negocio en las Empresas de Telecomunicaciones (pp. 1-13). Cuba. 
Serna Gómez, H. (1997). Gerencia estratégica: Planeación y gestión. Ediciones Uniandes. ISBN: 41000

Software Engineering Institute. (2010). CMMI Product Team, CMMI for Services. Version 1.3. Carnegie Mellon University

Software Engineering Institute. (2012). Software Engineering Institute. Recuperado el 23/04/2012 en: http://www.sei.cmu.edu/

Sparano Rada, H. (2011). Impacto de las áreas de conocimiento de la administración de proyectos a través del PMBOK 2008. ISSN 1692-8563, Vol. 9, №. 1, 2011, págs. 64-73. Recuperado el 18/04/2012 en: http://dialnet.unirioja.es/servlet/articulo?codigo $=3797760$

Sylvester, D. (2011). ISO 38500-Why Another Standar?. Recuperado el 25/03/2011 en http://www.isaca. org/Knowledge-Center/Documents/COBIT-Focus-ISO-38500-Why-Another-Standard.pdf.

Toomey, M. (2009). A Framework for Governance and Management of IT. Recuperado el 13/01/2012 en: http://www.infonomics.com.au/Web\%20Content/Documents/The_Infonomics_Letter_May_2009.pdf

Turbitt, K. (2006). ISO 20000: ¿qué deben hacer las organizaciones? bmcSoftware Activate Business with the power of IT.

Weill, P., y Ross, J. (2004). IT Governance. How top performers manage IT decision rights for superior results. Boston, MA.: Harvard Business School Press.

Zamora Jiménez J. (27 de Octubre de 2010). Análisis de las herramientas de gobierno de las TI y propuesta de un diseño para una PYME basándose en ITIL y COBIT. (pp. 9). Madrid, España. 


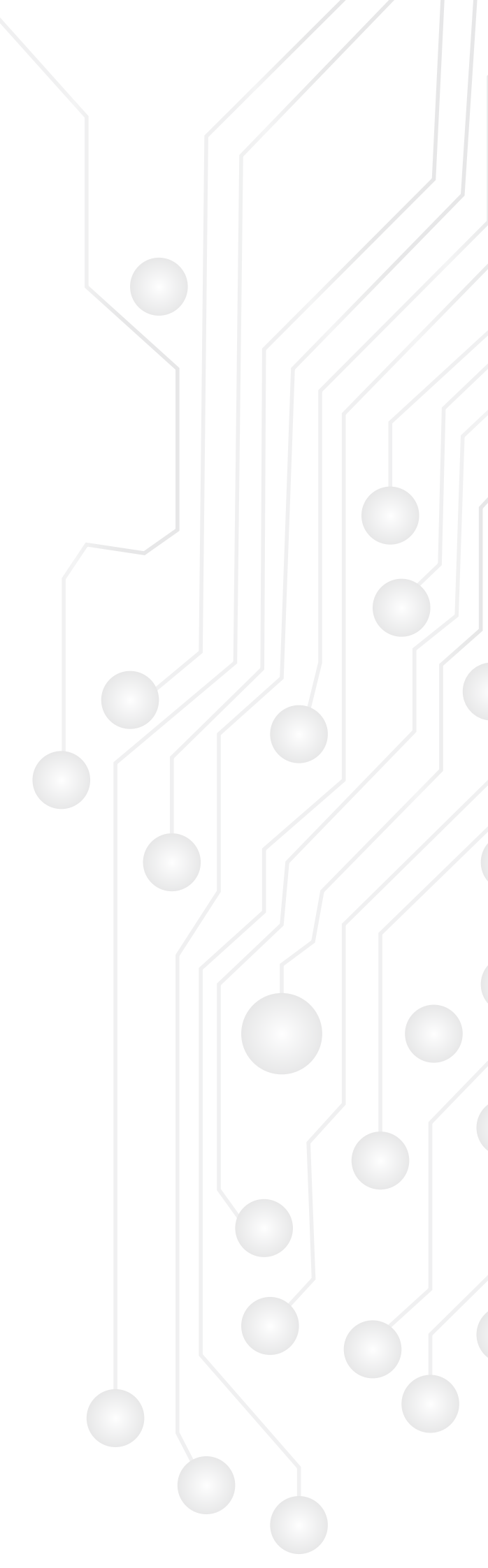

